\title{
BMJ Open Trends from the surveillance of suicidal behaviour by the Belgian Network of Sentinel General Practices over two decades: a retrospective observational study
}

\author{
Nicole Boffin, Sarah Moreels, Viviane Van Casteren
}

To cite: Boffin N, Moreels S, Van Casteren V. Trends from the surveillance of suicidal behaviour by the Belgian Network of Sentinel General Practices over two decades: a retrospective observational study. BMJ Open 2015;5: e008584. doi:10.1136/ bmjopen-2015-008584

- Prepublication history and additional material is available. To view please visit the journal (http://dx.doi.org/ 10.1136/bmjopen-2015008584).

Received 4 May 2015 Revised 15 October 2015 Accepted 2 November 2015

CrossMark

Scientific Institute of Public Health, OD Public Health and Surveillance, Brussels, Belgium

Correspondence to Dr Nicole Boffin; nicole.boffin@wiv-isp.be

\section{ABSTRACT}

Objectives: First, we describe trends in characteristics of suicidal events using new (2011-2012) and previous (1993-1995, 2000-2001 and 2007-2008) data reported by the Belgian Network of Sentinel General Practices (SGP); second, we examine patient age-related trends in on-site attendance of sentinel general practitioners (GPs) as first professional caregivers following suicidal behaviour; third, we investigate the accuracy of suicide incidence estimates derived from the SGP data.

Design: Retrospective observational study.

Setting: General practices from the nationwide representative Belgian Network of SGP.

Outcome measures: Patient gender and age, suicide methods, whether the patient was new, whether the GP was the first caregiver on-site, and the outcome of the suicidal behaviour (fatal or not) were recorded on standard registration forms. The accuracy of suicide incidence estimates was tested against suicide mortality data.

Results: Over the four time periods, 1671 suicidal events were reported: 275 suicides, 1287 suicide attempts and 109 events of suicidal behaviour of unknown outcome. In 2011-2012, sentinel GPs' onsite attendance following the suicidal behaviour of patients $<65$ years had continued to decrease (from $71 \%$ in $1993-1995$ to $58 \%$ in $2000-2001,39 \%$ in 2007-2008 and 25\% in 2011-2012). In 2011-2012, it had also decreased steeply in the population $\geq 65$ years (from 70\% in 1993-1995, 76\% in 2000-2001 and $79 \%$ in $2007-2008$ to $35 \%$ in $2011-2012$ ). No significant differences were found between the SGPbased suicide incidence estimates for 2011-2012 and the available suicide mortality rates for people $<65$ and $\geq 65$ years.

Conclusions: GPs' on-site attendance as first professional caregivers following suicidal behaviour continues to decline since 2011-2012 also in the population $\geq 65$ years. Unawareness of patients' suicidal behaviour endangers both care for surviving patients and the completeness of SGP surveillance data. Yet, the incidence of suicide for 2011-2012 was estimated accurately by the SGP.

\section{Strengths and limitations of this study}

- This is the first study of general practitioners (GPs) on-site attendance as first professional caregivers following suicidal behaviour. We found that Belgian GPs are moving back from the first line of care for patients who have engaged in suicidal behaviour.

- We found that characteristics of suicidal events were missing, that is, not reported by the Sentinel General Practices (SGP), largely due to their non-applicability or to loss of patients who were never seen before. This data-derived knowledge about item non-response may be considered an indicator of data quality.

- Since suicidal behaviour is a relatively rare event, Cls are wide and some characteristics of suicidal events are described broadly.

- The measure recording whether a patient was new was not included every year, and also varied over time. We did not examine the accuracy of the SGP estimates of the incidence of suicide attempts in the population.

\section{INTRODUCTION}

The majority of people who seek care for mental health problems consult a primary care provider and general practitioners (GPs) have become the vanguard in the fight against suicide. ${ }^{12}$ On average, $45 \%$ of people who died by suicide had contact with a primary care provider in the month before their death. ${ }^{3}$ Improving depression recognition and suicide risk evaluation among GPs is therefore considered an important component of suicide prevention. ${ }^{4}$ GPs play a key role in Belgian healthcare, even though patients are basically free to consult any care provider. Overall, $95 \%$ of the general population in Belgium has a regular GP and around half of all general practice patients have a 'general health record', that is, a 
GP-held record of all their medical information including reports from other care providers. ${ }^{5}{ }^{6}$ Since 1982, the surveillance of suicidal behaviour, that is, suicide (fatal outcome) and suicide attempts (non-fatal outcome), has been carried out by the Belgian Network of Sentinel General Practices (SGP). This surveillance includes all events of suicidal behaviour that the sentinel GPs are confronted with, including the suicidal behaviour of new patients seen (for the first time) during out-of-hours care, as well as the suicidal behaviour of their own patients as reported by other care providers. The surveillance provides preliminary data that are complementary to national mortality statistics and is one of the only sources to monitor suicide attempts in the Belgian population.

In this paper, we describe trends over the past four time periods in the light of findings from three previous periods. First, we found that sentinel GPs' on-site attendance as first professional caregivers (called out) following the suicidal behaviour of patients $<65$ years had declined from $71 \%(1993-1995)$ to $58 \%$ (2000-2001) and $39 \%$ (2007-2008). ${ }^{7}$ Among people $\geq 65$ years, these rates have remained at the same average level of $74 \%$ since 1993-1995. This trend makes sense, as the use of hospital emergency services for standard and less urgent problems by self-referred patients in Belgium ${ }^{8}$ may be less of a problem among older people. Older people relatively have more consultations with their usual GP, ${ }^{9}$ more GP contacts and home visits, ${ }^{5}$ and relatively more older people have a general health record. ${ }^{6}$ It is likely that sentinel GPs receive late reports or no reports at all from other care providers about the suicidal behaviour of their own patients to whom they were not called out and, as a result, sentinel GPs do not report these events on their weekly forms. GPs' unawareness of suicidal behaviour among practice patients impedes appropriate care for surviving patients and also jeopardises the completeness of SGP reporting.

Second, we found that the incidence of suicide in the Belgian population was underestimated for 2007-2008 in comparison to the gold standard, the suicide mortality data based on death certificates for 2007-2008 (available as of February 2013). Yet, the SGP-based incidence of suicide among people $\geq 65$ years for 2007-2008 did not differ significantly from the national suicide mortality rate in the same period. ${ }^{10}$ Previously, the SGP-based suicide incidence estimates had been comparable to the mortality statistics. ${ }^{11}{ }^{12}$ No gold standard exists to which the SGP-based incidence estimates for suicide attempts can be compared. However, the decrease in the SGP-based incidence estimates for suicide attempts in 2007-2008 is not in line with the absence of any significant trend between 1993-1995 and 2000-2001.

In short, the overall aim of this paper is to study our assumption that the underestimated incidence for 20072008 is partly explained by sentinel GPs' unawareness, and thus non-registration, of the suicidal behaviour by patients <65 years that was not seen by the GPs.
Therefore, we first describe trends in patient gender and age, suicide methods, whether the patient was new, whether the GP was the first caregiver on-site and the outcome of the suicidal behaviour (fatal or not). The aforementioned variables are also examined where missingness exceeds $1 \%$. Second, we examine patient age-related trends in sentinel GPs' on-site attendance as first professional caregivers following suicidal behaviour of patients. Third, we investigate the accuracy of the SGP-based estimates of suicide incidence in the population over four time periods relative to suicide mortality data.

\section{METHODS}

\section{Data source}

The Belgian network of SGP was developed in 1979 by the Belgian Institute of Public Health drawing on experiences of sentinel surveillance in the UK and the Netherlands. ${ }^{13}$ The network comprises approximately 150 general practices with one or more sentinel GPs who purposively record routine clinical care data for the surveillance of specific health problems or care delivery. The gender-age distributions of sentinel and nonsentinel GPs by region are comparable and the network covers between $1.4 \%$ and $1.8 \%$ of the Belgian population throughout all regions. ${ }^{14}$ Since Belgian GPs do not serve a defined practice population, the size of the SGP patient population is estimated by applying the ratio of patient contacts in the entire Belgian population to the sum of weekly patient contacts in the network.

\section{Data collection}

The data were reported by the SGP on weekly baseline forms and on follow-up forms either 2 weeks (19932008) or 4 weeks (2011-2012) after the suicidal event. Suicide and suicide attempt were briefly defined as 'an act of self-inflicted injury or self-poisoning (excess of the generally recognised therapeutic dosage in the case of self-poisoning with drugs) with fatal (suicide) or nonfatal outcome (suicide attempt)'. Any suicidal behaviour which ultimately resulted in death after 2-4 weeks was considered a suicide. Follow-up forms were used to report the outcome of the suicidal behaviour, that is, survival or not, and, if necessary, to complete data that were missing on the baseline form. Since 2011, the SGP programme staff has been sending lists of recorded suicidal events to the SGP on a regular basis, even when no events were reported, and requesting that they be returned after correction, completion or confirmation of their content.

\section{Variables and measurements}

Five variables, including patients' gender and age, are the common core of the baseline forms that were used in the four periods. Specific suicide methods listed on the form were regrouped into a single dummy variable with a value of one for violent methods, combined or 
not with non-violent methods, and a value of zero for non-violent methods only. ${ }^{15}$ Non-violent methods include self-poisoning by pharmacological agents, gases or other toxic substances (free text space). Violent methods include hanging, drowning, firearm, wrist cutting, jumping from a height, crashing a car or jumping or lying in front of a train. Other suicide methods reported as free text were categorised according to ICD-10, for example, suffocation and strangulation were categorised as hanging. The measure whether the patient was new has changed over time. In 19931994, the sentinel GPs were requested to tick a box if the patient was never seen before. We did not use the information from another box to tick if the patient was seen out-of-hours. In 1995, no information was asked concerning this subject. In 2000-2001, sentinel GPs were asked to record only whether the patient was seen during out-of-hours care, not whether he or she was a new patient. In 2011-2012, a box was to be ticked if the patient 'was never seen before/during out-of-hours care'. We show descriptive statistics on this measure for 2000-2001 (see online supplementary table S1), but in the analysis (see next subsection) we only use data from the first and the last periods for the measure labelled as 'the patient was new'. The type of professional who was the first to provide care to the patient was recoded into a second dummy variable with a value of one when the sentinel GP had been the first professional caregiver (called out). Alternatives to the GP were immediate hospitalisation, ambulatory mental health provision or other professional care (free text space).

\section{Analysis}

The characteristics of suicidal events by period were reported with $95 \%$ binomial proportion CIs. Data from 2000 to 2001 for the measure whether the patient was new were only used in univariate analysis. A score test for trend of odds was used to detect trend in the characteristics of suicidal events over time. Significant time trends (except GPs' on-site attendance, see further) were examined by multiple logistic regression adjusted for age and gender.

Univariate associations between missingness of data exceeding $1 \%$ and characteristics of suicidal events were examined by a $\chi^{2}$ test. Significant variables $(p<0.05)$ were included in multiple logistic regression models for prediction of missingness. The measure whether the patient was deceased on arrival of the sentinel GP was only included in the univariate analysis of missingness, but not in any multiple logistic regression model because of collinearity with survival. This subject was reported in the first three periods but not in the fourth period.

Determinants of GPs' on-site attendance as first caregivers following suicidal behaviour were examined by multiple logistic regression. Variables in the full model included age, gender, SGP region and suicide methods which were significantly $\left(\chi^{2}\right.$ test $\left.\mathrm{p}<0.05\right)$ associated with
GPs' on-site attendance. Interaction effects between independent variables were tested in all models. A generalised estimating equation approach was used to account for the clustering of data within general practices.

Annual incidence estimates were calculated by dividing the number of events by the sum of the person years covered by the SGP in the period concerned. CIs for incidence rates were calculated using a Poisson distribution. The accuracy of the SGP estimates of suicide incidence for 2000-2001 and 2011-2012 was measured for Flanders alone, as mortality statistics for the entire country were not yet available for those years (assessed 15 March 2015). In 2013, Flanders was inhabited by $57.5 \%$ of the Belgian population and was therefore the largest region in Belgium. ${ }^{16}$ We used a two-sample test of proportion to measure the accuracy of the SGP estimates. Data were analysed using Stata V.10 and V.13.

\section{RESULTS}

Trends in key characteristics of suicidal events and missing data

A total of 1671 suicidal events were reported over the four time periods. Among these were 275 suicides, 1287 suicide attempts and 109 events of suicidal behaviour of unknown outcome (fatal or non-fatal) (see online supplementary table S1).

\section{Trends in key characteristics of suicidal events}

Two characteristics showed a significant score test for trend of odds. First, the proportion of violent methods was found to have increased in the total sample and in the sample of suicide attempts. Violent methods were used in 553 of $1657(33.4 \%)$ events, and combined with non-violent methods in 42 of $553(7.6 \%)$ events. Logistic regression adjusted for age and gender revealed that the odds of violent methods were not significantly associated with time period in the sample of suicides, suicide attempts or overall (data not shown). Second, sentinel GPs' on-site attendance was found to have decreased in the samples of suicides and suicide attempts (see Sentinel GPs' on-site attendance section). The proportion of patients seen for the first time in 2011-2012 was the same as that seen in 1993-1995. The increasing proportions of men and people $<65$ years over time were both borderline significant in the total sample, but no trend was seen in the samples of suicides or suicide attempts. No time trend was observed in survival rates.

\section{Missing data exceeding 1\%}

No variables were independently associated with missing age for 25 of $1671 \quad(1.5 \%)$ events (see online supplementary table S2). However, age was more often missing when the patient was seen for the first time than when the patient had been seen before $(p<0.05)$.

Data on the first professional caregiver on-site were more often missing for suicides than for suicide attempts 
$(\mathrm{p}<0.001)$. The first three surveillance studies showed that data on the first professional caregiver were more often missing for patients who were already deceased on the arrival of the sentinel GP than for patients who were still alive $(p<0.001)$. Fatal outcome, that is, suicide, was the only variable independently associated with missing data for the first professional caregiver (OR 11.2; 95\% CI 7.3 to 17.2 ).

The outcome of the suicidal behaviour was more often missing for new patients than for regular practice patients $(p<0.001)$. Seeing the patient for the first time (versus otherwise) was the only variable independently associated with unknown outcome of suicidal behaviour (OR 11.4; 95\% CI 4.8 to 27.1).

\section{Sentinel GPs' on-site attendance}

In 2011-2012, sentinel GPs' on-site attendance following the suicidal behaviour of patients $<65$ years continued to decrease and it had also dropped in the population $\geq 65$ years (figure 1 ).

The overall model of factors associated with sentinel GPs' on-site attendance following suicidal behaviour revealed a significant interaction between time period and age group $(\mathrm{p}=0.026)$ (table 1$)$.

Modelling the factors on populations split by age group shows a decline in sentinel GPs' on-site attendance following the suicidal behaviour of patients $<65$ years in every period compared to the first surveillance period, while in the population $\geq 65$ years a significant decline was seen only in the most recent period. When the most recent period (2011-2012) was contrasted to the three previous periods, no significant interaction was observed between time period and age group in the overall model.

\section{Accuracy of SGP suicide incidence estimates}

In 2011-2012, there were no significant differences between the SGP estimates of suicide incidence among people $<65$ years and people $\geq 65$ years in Flanders and the corresponding suicide mortality rate (table 2 ).

\section{a3-5 $=2000-1 \quad$ 2007-8 $\square 2011-2$}

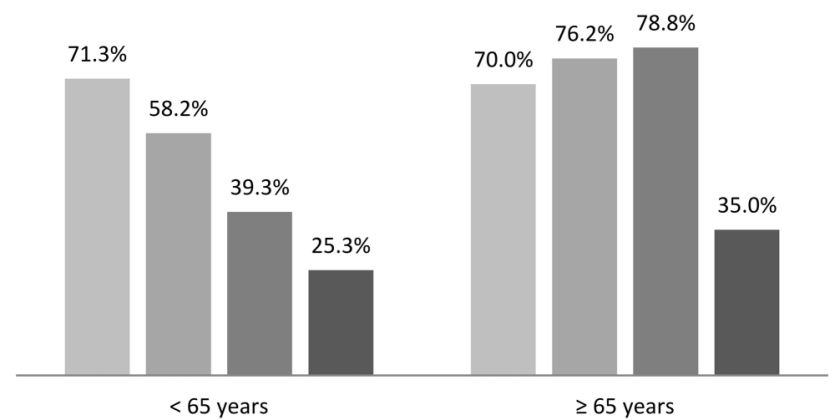

Figure 1 Percentages of suicidal events to which sentinel general practitioners were called out as first professional caregivers over four time periods by patient age groups $(<65$ and $\geq 65$ years).
Table 1 Results of marginal generalised estimating equation models for association between sentinel general practitioners' on-site attendance as first professional caregivers following suicidal behaviour and time period by patient age groups

\begin{tabular}{ll} 
Patients $<65$ years $(\mathbf{n = 1 3 4 3 )}$ & \\
\hline Period & Adjusted OR $(\mathbf{9 5 \%} \mathbf{C l})^{\star}$ \\
\hline $1993-1995$ & reference \\
$2000-2001$ & $0.57(0.42$ to 0.76$)$ \\
$2007-2008$ & $0.27(0.19$ to 0.40$)$ \\
$2011-2012$ & $0.15(0.10$ to 0.23$)$
\end{tabular}

Patients $\geq 65$ years $(n=173)$

\begin{tabular}{cl}
\hline Period & Adjusted OR $(95 \% \mathbf{C l})^{\star}$ \\
\hline $1993-1995$ & reference \\
$2000-2001$ & $1.95(0.71$ to 5.38$)$ \\
$2007-2008$ & $1.77(0.63$ to 4.96$)$ \\
$2011-2012$ & $0.26(0.12$ to 0.57$)$
\end{tabular}

${ }^{*}$ Adjusted for Sentinel General Practices region, patient gender and suicide method (that is, violent methods by hanging, jumping from a height, crashing a car and jumping or lying in front of a train and self-poisoning by toxic agents other than pharmacological agents and gases).

\section{DISCUSSION}

Surveillance of suicidal behaviour by the Belgian Network of SGP over two decades shows that sentinel GPs' on-site attendance as first professional caregivers following suicidal behaviour continued to decline in 2011-2012. For the first time, this decline was seen in the population $<65$ years, as well as in the population $\geq 65$ years. The SGP estimates of suicide incidence in Flanders for 2011-2012 were found to be accurate for both age groups. We showed that missing data on age and survival were predominantly attributable to nonregular or new patients. We equally found that data on the first caregiver were missing mainly because of nonapplicability, that is, no care was given to patients who died on the spot.

Since the SGP network is representative of the national workforce, this study demonstrates that Belgian GPs are moving back from the first line of care for patients who have engaged in suicidal behaviour. Using the military metaphor again, the Belgian 'vanguards in the fight against suicide' are falling back from the frontline. This trend may be attributed to the increasing use of hospital emergency services, now also among people $\geq 65$ years. Yet, as described in the introduction, GPs are the care providers par excellence for older people. This was confirmed by our study of suicide death certificates in Flanders during 6 months in 2009-2010, showing that GPs completed relatively more suicide death certificates for people $\geq 65$ years compared to non-GPs. ${ }^{10}$ In contrast to older people, less young and middle-aged people have a regular GP. ${ }^{5}$ Moreover, international studies found that few young men consult a GP in the period before suicide and the primary care contact rate in the 
Table 2 Sentinel General Practices (SGP)-based estimates of suicide incidence among inhabitants of Belgium or Flanders * and suicide mortality data by population age groups over four periods

\begin{tabular}{|c|c|c|c|}
\hline \\
\hline \multicolumn{2}{|c|}{$\begin{array}{ll}1993-1995 \dagger & \text { SGP estimates Belgium }\end{array}$} & \multicolumn{2}{|c|}{ Annual incidence per 100000 inhabitants and $\mathbf{9 5 \%} \mathrm{Cl}$} \\
\hline$<65$ years & \multicolumn{2}{|c|}{$\begin{array}{l}\text { SGP estimates Belgium } \\
19.5(15.3 \text { to } 24.4)\end{array}$} & 0.802 \\
\hline \multirow{2}{*}{$\begin{array}{l}\geq 65 \text { years } \\
\text { Total }\end{array}$} & 26.6 (16.0 to 41.5$)$ & 34.6 (33.0 to 36.3 ) & \multirow[t]{2}{*}{0.249} \\
\hline & $20.6(16.6$ to 25.1$)$ & 21.4 (20.9 to 21.9$)$ & \\
\hline \multirow{4}{*}{$\begin{array}{l}2000-2001 \dagger \\
<65 \text { years } \\
\geq 65 \text { years } \\
\text { Total }\end{array}$} & SGP estimates Flanders & \multicolumn{2}{|l|}{ Suicide mortality Flanders } \\
\hline & 18.5 (12.3 to 26.7$)$ & $18.4(17.5$ to 19.2$)$ & 0.979 \\
\hline & 26.5 (11.4 to 52.2$)$ & 24.8 (22.7 to 27.1$)$ & \multirow{2}{*}{0.856} \\
\hline & $19.8(13.9$ to 27.4$)$ & 19.5 (18.7 to 20.3$)$ & \\
\hline \multirow{2}{*}{$\begin{array}{l}2007-2008 \dagger \\
<65 \text { years }\end{array}$} & SGP estimates Belgium & Suicide mortality Belgium & \\
\hline & $12.1(8.5$ to 16.7$)$ & $17.4(16.8$ to 18.0$)$ & 0.028 \\
\hline \multirow{2}{*}{$\begin{array}{l}\geq 65 \text { years } \\
\text { Total }\end{array}$} & 24.4 (13.7 to 40.2$)$ & 21.4 (19.9 to 22.9$)$ & \multirow{2}{*}{0.613} \\
\hline & $14.2(10.6$ to 18.6$)$ & $18.1(17.5$ to 18.7$)$ & \\
\hline \multirow{2}{*}{$2011-2012$} & SGP estimates Flanders & Suicide mortality Flanders & \\
\hline & $17.7(12.0$ to 25.2$)$ & $16.6(15.8$ to 17.4$)$ & \multirow{3}{*}{$\begin{array}{l}0.714 \\
0.95\end{array}$} \\
\hline \multirow{2}{*}{$\begin{array}{l}\geq 65 \text { years } \\
\text { Total }\end{array}$} & 22.7 (10.4 to 43.2$)$ & 23.2 (21.3 to 25.2$)$ & \\
\hline & $18.6(13.3$ to 25.4$)$ & $17.8(17.1$ to 18.5$)$ & \\
\hline \multicolumn{4}{|c|}{$\begin{array}{l}\text { *Statistics available at http://www.zorg-en-gezondheid.be/Cijfers/Sterftecijfers/Statistiek-van-de-doodsoorzaken/ and https://spma.wiv-isp.be/ } \\
\text { SitePages/Home.aspx. } \\
\text { †Figures for 1993-1995, 2000-2001 and 2007-2008 were published previously. }{ }^{17} \\
\text { fTwo-sample test of proportion. }\end{array}$} \\
\hline
\end{tabular}

month preceding suicide is much lower among people $<55$ years. ${ }^{3}{ }^{18}$ As a result, non-consultation of people at risk limits both the scope of suicide prevention in general practice and the inference of the SGP results for the general population.

In 2011-2012, the incidence of suicide was again estimated accurately by the SGP, despite the growing trend of suicides for which the sentinel GPs were not called out across the two age groups. Two interventions may have improved the completeness of SGP reporting of suicidal behaviour. First, we reported the underestimation of the incidence of suicidal behaviour in 2007-2008 in the SGP newsletter. Second, the SGP programme staff began sending lists of recorded events to the SGP on a regular basis in order to enhance the completeness of their reporting. The completeness of hospitals' and other health services' reporting of suicidal behaviour to GPs may also have improved. The Flemish Ministry of Health commissioned the development and implementation of an Instrument for Psychosocial Evaluation and Care for Suicide Attempters (IPEO), which included training hospital staff, developing a clinical pathway in hospitals and ensuring continuity of care after discharge by involving GPs and community mental health centres. ${ }^{19}$ Since 2013, the SGP surveillance programme has been monitoring the information received by GPs from hospitals about suicide attempters in their practice.

As far as we know, this is the first study of GPs' on-site attendance as first professional caregivers following suicidal behaviour. Surveillance by a network of sentinel GPs is a simple, flexible and affordable method for collecting original data on health problems in general practice and their management by GPs. Our finding that characteristics of suicidal events were missing mainly because of their unavailability or non-applicability, not because of non-understanding or non-compliance by the sentinel GPs, may be considered an indicator of data quality. ${ }^{20}$ This study shows, however, that this type of surveillance is less well suited to monitor the occurrence of rare events, such as suicidal behaviour. As a result, CIs for epidemiological estimates are wide and the characteristics of suicidal events are grouped into broader categories. Another study weakness lies in its rough measures. The heterogeneity of violent methods and the change of the variable describing whether the patient was new or was seen (for the first time) during out-of-hours care exemplify this weakness. Finally, we did not examine the accuracy of the SGP estimates of the incidence of suicide attempts in the population. When using IPEO-based data from accident and emergency departments in Flemish general hospitals, estimates of the incidence of suicide attempts in Flanders for 2011$2012(183 / 100000$ in 2011 and 162/100 000 in 2012) were found to be much higher than the SGP estimates for 2011-2012 (57/100 000). ${ }^{21}$ The discrepancy between these sources should gradually decrease if IPEO is successful in involving GPs in follow-up care for suicide attempters. SGP surveillance data for 2013-2014 will provide the first data to examine this assumption.

\section{CONCLUSION}

Over two decades, GPs' on-site attendance as first professional caregivers following suicidal behaviour of patients continues to decline, no longer in the population $<65$ years alone but since 2011-2012 also in the population 
$\geq 65$ years. This trend endangers both care for surviving patients and the completeness of data. Yet, the most recent SGP estimates of suicide incidence in Flanders for 2011-2012 were accurate for both population age groups. Since 2013, the SGP has been monitoring the information received by GPs from hospitals about practice patients who receive hospital care for self-inflicted injuries.

Acknowledgements All GPs from the Belgian Network of Sentinel General Practices are gratefully acknowledged.

Contributors NB, SM and VVC were involved in the study conception, study design, data collection, interpretation of results and finalisation of the manuscript. NB was also involved in the data analysis and writing of the manuscript.

Funding Flemish Agency for Care and Health and the Wallonia-Brussels Federation

Competing interests None declared.

Ethics approval Ethical Committees of the Scientific Society of Flemish GPS and the Catholic University of Louvain.

Provenance and peer review Not commissioned; externally peer reviewed.

Data sharing statement No additional data are available.

Open Access This is an Open Access article distributed in accordance with the Creative Commons Attribution Non Commercial (CC BY-NC 4.0) license, which permits others to distribute, remix, adapt, build upon this work noncommercially, and license their derivative works on different terms, provided the original work is properly cited and the use is non-commercial. See: http:// creativecommons.org/licenses/by-nc/4.0/

\section{REFERENCES}

1. Bostwick JM, Rackley S. Addressing suicidality in primary care settings. Curr Psychiatry Rep 2012;14:353-9.

2. Bruffaerts R, Bonnewyn A, Van Oyen $\mathrm{H}$, et al. [Patterns of service use for mental health disorders in Belgium. Results of the European Study on Epidemiology of Mental Disorders ESEMeD]. Rev Med Liege 2004;593:136-44.

3. Luoma JB, Martin CE, Pearson JL. Contact with mental health and primary care providers before suicide: a review of the evidence. $A m$ J Psychiatry 2002;159:909-16.

4. van der Feltz-Cornelis CM, Sarchiapone M, Postuvan V, et al. Best practice elements of multilevel suicide prevention strategies. Crisis 2011;32:319-33.

5. Belgian Health Interview Survey-Interactive analysis HISIA. Brussels: Scientific Institute of Public Health. https://hisia.wiv-isp.be/ (accessed 15 Mar 2015).
6. Meeus P. [General practice performance. A check up]. Brussel: RIZIV 2012.

7. Boffin N, Bossuyt N, Vanthomme K, et al. Declining rates of suicidal behavior among general practice patients in Belgium: results from sentinel surveillance between 1993 and 2008. Arch Suicide Res 2011;15:68-74.

8. Philips $\mathrm{H}$, Mahr D, Remmen $\mathrm{R}$, et al. Predicting the place of out-of-hours care-a market simulation based on discrete choice analysis. Health Policy 2012;106:284-90.

9. De Maeseneer JM, De Prins LF, Heyerick JP, et al. [The loyalty of Belgian patients to their family practitioner]. Ned Tijdschr Geneeskd 1994;138:2649-54

10. Boffin N, Bossuyt N, Vanthomme K, et al. Are Belgian general practitioners more aware of suicides in older ( $\geq 65$ years) than in young and adult persons? A multimethod study. Surveillance studies of suicidal behavior and depression by the Belgian Network of Sentinel General Practices [PhD-thesis]. Ghent University, 2013:75-90.

11. Bossuyt N, Van Casteren V. Epidemiology of suicide and suicide attempts in Belgium: results from the sentinel network of general practitioners. Int J Public Health 2007:52:153-7.

12. Van Casteren V. [Surveillance of suicide and suicide attempts by a network of sentinel general practices in Belgium. Comparison between 1993-1995 and 1990-1992]. Tijdschr Geneeskd 1999;55:83-7

13. Van Casteren V. Thirty years Registration Network of Sentinel General Practitioners. Arch Public Health 2009;67(Suppl. 2):3-15.

14. Boffin N, Moreels S, Van Casteren V. The Belgian Network of Sentinel General Practices between 2007 and 2012: a short report Scientific Institute of Public Health, OD Public Health and Surveillance. Brussels: Health Services Research 2013.

15. Denning DG, Conwell Y, King D, et al. Method choice, intent, and gender in completed suicide. Suicide Life Threat Behav 2000;30:282-8.

16. Standardized Procedures for Mortality Analysis-Belgium. Brussels: Scientific Institute of Public Health. https://spma.wiv-isp.be (accessed 15 Mar 2015).

17. Boffin N. Surveillance studies of suicidal behavior and depression by the Belgian Network of Sentinel General Practices [PhD thesis]. Ghent University 2013.

18. Stanistreet $\mathrm{D}$, Gabbay $M B$, Jeffrey $\mathrm{V}$, et al. The role of primary care in the prevention of suicide and accidental deaths among young men: an epidemiological study. $\mathrm{Br} J$ Gen Pract 2004;54501:254-8.

19. Vanhove R, Van de Craen T, Portzky G, et al. [Good practices in the care for suicide attempters: evaluation of the Project Integrated Care for Suicide Attempters" in general hospitals in Flanders Belgium]. Tijdschr Geneeskd 2013;69:657-62.

20. Weiskopf NG, Rusanov A, Weng C. Sick patients have more data: the non-random completeness of electronic health records. AMIA Annu Symp Proc 2013;2013:1472-7.

21. De Jaegere E, Vancayseele N, Portzky G, et al.[Epidemiology of suicide attempts in the Flemish Region. Report 2012]. Gent: Eenheid voor Zelfmoordonderzoek, 2013. 